\title{
Nonlinear magneto-optic polarization rotation with intense laser fields
}

\author{
Paul S. Hsu, ${ }^{1, *, \dagger}$ Anil K. Patnaik, ${ }^{1,2, \dagger}$ and George R. Welch ${ }^{1}$ \\ ${ }^{1}$ Department of Physics, Texas A\&M University, College Station, Texas 77843-4242, USA \\ ${ }^{2}$ Department of Physics, Wright State University, Dayton, Ohio 45435, USA
}

(Received 23 August 2008; published 14 November 2008)

\begin{abstract}
We have studied the nonlinear Faraday effect with intense linear polarized light in an optically thick atomic rubidium vapor. We demonstrate that the polarization rotation rate (rotation angle per unit magnetic field, in the limit of low field) has a maximum value as the intensity and density are increased. We also show that the optimal sensitivity of an optical magnetometer based on this system reaches a saturation value as the intensity and density are increased.
\end{abstract}

DOI: $10.1103 /$ PhysRevA.78.053817

PACS number(s): 42.50.Gy, 07.55.Ge, 42.50.Nn, 32.60.+i

\section{INTRODUCTION}

There is immense interest in improving the sensitivity of polarization spectroscopy measurements $[1,2]$, both for fundamental and practical reasons such as optical magnetometry [3]. The sensitivity of optical pumping magnetometers (OPMs) has already achieved $10^{-9} \mathrm{G} / \sqrt{\mathrm{Hz}}$ under laboratory conditions $[4,5]$. Also, nonlinear magneto-optic rotation has been used in magnetometry to reach very high sensitivity [6]. In such devices, the Zeeman level shift measurements are based on light absorption [7], but the sensitivity is limited if the absorption is strong. Scully and Fleischhauer et al. [8,9] described a high-sensitivity optical magnetometer based on electromagnetically induced transparency (EIT), where the high dispersion at an EIT resonance can dramatically improve the sensitivity of magnetic field measurements by suppression of the absorption via quantum interference.

We describe nonlinear magneto-optic polarization rotation as follows. Consider two circularly polarized electromagnetic waves near atomic resonance in a $\Lambda$ configuration as shown in Fig. 1. When the two frequencies are in Raman (two-photon) resonance, a "dark state" is created that is associated with electromagnetically induced transparency [10]. The atoms are optically pumped into a transparent coherent superposition of ground-state Zeeman sublevels that is accompanied by very steep dispersion. This dispersion gives rise to such effects as enhanced index of refraction [11] and ultraslow light [12]. Therefore, even a small shift of magnetic sublevels can result in a large change in the refractive indices for the two circular components, so that they acquire different optical phase shifts after traversing the length of the cell. This phase difference results in rotation of the polarization of linear polarized light exiting the cell with respect to the direction at which it entered. This effect is known as nonlinear magneto-optical rotation (NMOR). If $\chi_{+}\left(\chi_{-}\right)$represents the susceptibility of the birefringent medium corresponding to the right (left) circular component of the probe, the rotation angle, for small absorption, is given by

\footnotetext{
*Present address: Air Force Research Laboratory, Propulsion Directorate, Wright-Patterson AFB, Ohio 45433.

†p-hsu@tamu.edu
}

$$
\phi=\frac{k_{p} l}{4 \epsilon_{0}}\left(\chi_{-}-\chi_{+}\right)
$$

where $k_{p}$ corresponds to the propagation vector of the probe and $l$ is the length of the medium along the direction of propagation.

The use of NMOR for magnetometry in optically thin and thick media has been extensively studied $[6,13,14]$. These works show that the ground-state coherence dephasing plays an important role in decreasing sensitivity. Various methods have been used to reduce the dephasing rate, such as highquality antirelaxation walls $[4,6]$ and buffer gas [14]. These methods can effectively increase the lifetime of ground-state coherence, greatly improving magnetic field sensitivity. For example, Ref. [6] demonstrates sensitivity of 3 $\times 10^{-12} \mathrm{G} / \sqrt{\mathrm{Hz}}$. Further high-sensitivity work has been done using optical pump-probe magnetometry $[15,16]$.

The usual limit on measurement of the smallest Zeeman level shift is determined by signal-to-noise ratio. It was pointed out by Fleischhauer et al. [9] that the limit of the detectable magnetic field shift is governed by two fundamental restrictions: photon counting error due to the vacuum fluctuation of the laser field (shot noise), and coupling the laser field to nonresonant levels (ac-Stark shifts). Compensation of ac-Stark shifts has been studied experimentally [17]. Based on the shot-noise limit the smallest detectable magnetic field $\delta B_{z}$, has been written as [6]

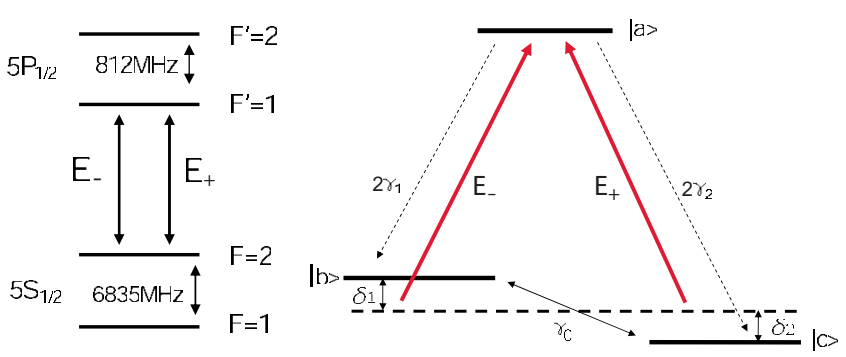

FIG. 1. (Color online) Energy levels of the ${ }^{87} \mathrm{Rb}$ atom used in the experiment, and corresponding simplified three-level $\Lambda$ diagram. 


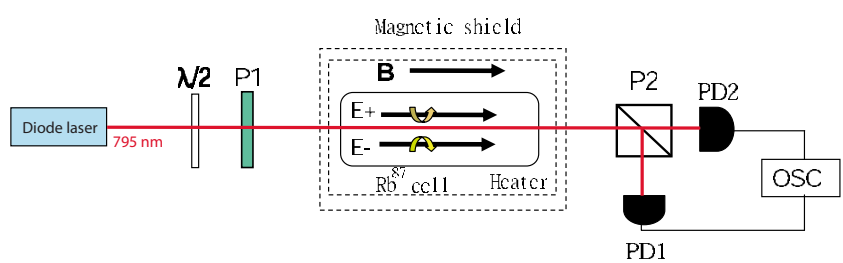

FIG. 2. (Color online) Diagram showing the experimental setup: $P 1$ is a polarizer; $\lambda / 2$ is a half-wave plate; $P 2$ is a polarized beam splitter cube; and OSC is an oscilloscope.

$$
\delta B_{z}=\frac{1}{\sqrt{N_{p h}}}\left(\frac{\partial \phi}{\partial B_{z}}\right)^{-1},
$$

where $N_{p h}$ is the number of photons counted, and $\frac{\partial \phi}{\partial B_{z}}$ is the rotation rate per unit magnetic field. Shot noise can be reduced by increasing the laser intensity, but this results in broadening of the EIT resonance, which decreases the rotation rate. In Ref. [9] it is proposed that the sensitivity of an optical magnetometer could be improved by simultaneously increasing both laser-field intensity and atomic density. This approach was studied for a limited range of laser intensity in [14]. It has also been shown that radiation trapping effects limit the polarization rotation by introducing a new type of dephasing [18].

In this paper, we show that there is a limiting value for the shot-noise-limited magnetic field sensitivity as the intensity and density are increased. The outline of the paper is as follows: The experimental setup and results are presented in Sec. II. In Sec. III, we describe our model system, derive dynamical equations, and show our numerical results. The physical explanations for the experimental results are given. A summary is presented in Sec. IV.

\section{EXPERIMENTAL SETUP AND MEASUREMENT}

Our experimental setup is shown in Fig. 2. A Toptica DLX 110 high-power tunable single mode diode laser is tuned to the $795 \mathrm{~nm} 5 S_{1 / 2}(F=2) \rightarrow 5 P_{1 / 2}\left(F^{\prime}=1\right)$ transition of the rubidium $D_{1}$ line, shown in Fig. 1. The laser propagates through a high-quality polarizer $P_{1}$, which produces linear polarization, then through a cylindrical glass cell of length $L=5.0 \mathrm{~cm}$ and diameter $D=2.5 \mathrm{~cm}$ containing isotopically enhanced ${ }^{87} \mathrm{Rb}$. The laser power can be controlled by a polarizer with a half-wave plate. To control the beam diameter, a beam expander may be placed after the polarizer. The atomic density of ${ }^{87} \mathrm{Rb}$ is controlled by the temperature of the coldest spot of the cell, which is installed in a temperature controlled double layer magnetic shield. A longitudinal magnetic field is created by a solenoid installed inside the magnetic shield. A polarization analyzer $P_{2}$ is placed after the cell and titled 45 degrees with respect to the polarizer. Photodiodes PD1 and PD2 detect the light from both channels of the analyzer, allowing simultaneous measurements of the polarization rotation angle $\phi$ and transmitted laser power.

In our experiment we consider the effect of different laser beam diameters and of different intensities of the laser. Because there is no buffer gas or wall coatings in our cell, changing the beam diameter changes the extent of the atomic
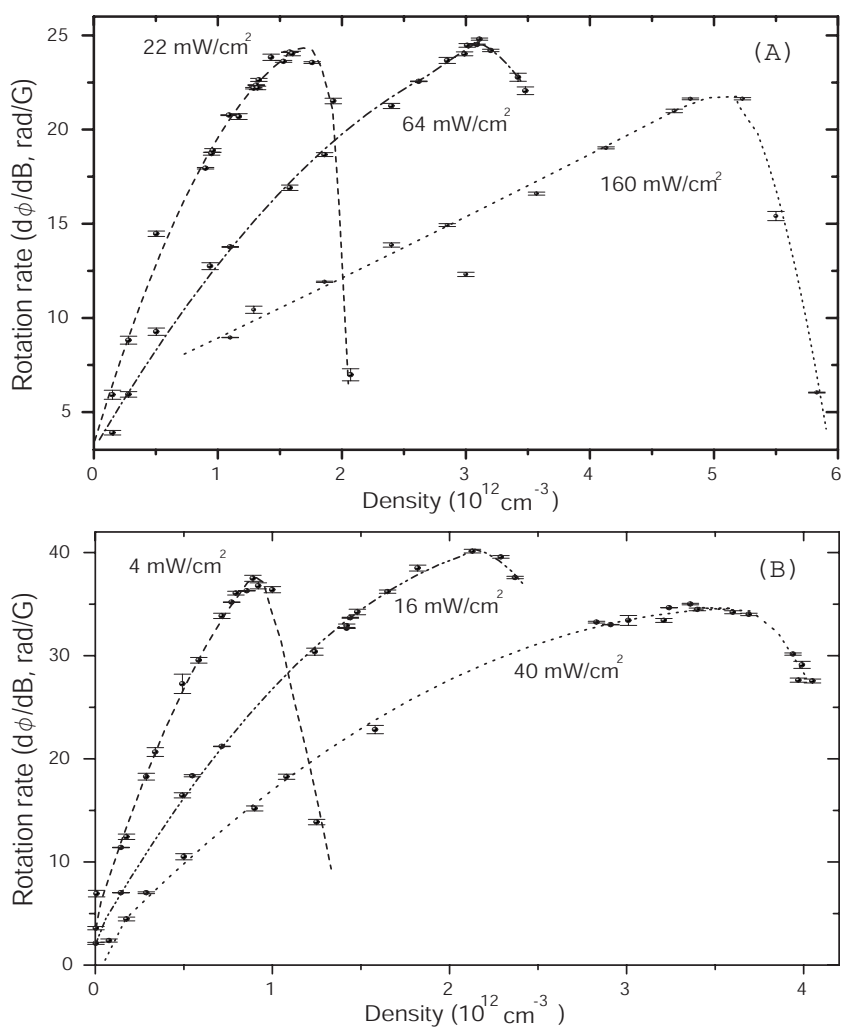

FIG. 3. Rotation rate $d \varphi / d B$ due to the nonlinear Faraday effect as a function of atomic density for the beam diameter (A) $d$ $=2 \mathrm{~mm}$ and (B) $d=4 \mathrm{~mm}$ for different intensities. The dashed lines are presented only to guide the eye.

interaction time with the laser (time of flight), and therefore changes the ground-state dephasing rate $\gamma_{0}$ [19]. Increasing the beam diameter leads to a smaller ground-state dephasing rate and should enhance the ground-state coherence resulting in better sensitivity.

Figure 3 shows the observed rotation rate as a function of density for two different beam diameters $(d=2 \mathrm{~mm}$ and $d$ $=4 \mathrm{~mm}$ ) and different laser intensities. The polarization rotation rate $d \phi / d B$ is obtained by measuring the polarization rotation for very small changes of magnetic field, such that changes in the polarization rotation are proportional to the changes in the magnetic field. The individual curves show that for a fixed intensity, the rotation rate increases with density, reaches a maximum value, and rolls off rapidly with increasing density. The maximum value increases with intensity. This trend of the individual curves has been explained [18]. However, the overall profiles of the rotation rate for larger intensities and densities from the linear region $(\Omega$ $\leqslant \gamma$ ) to the nonlinear region $(\Omega \gg \gamma)$ has not been previously explored, where $\Omega$ is the Rabi frequency of the applied laser with atomic transition.

For each value of the laser intensity, there is a specific density where the rotation rate is maximum. Figure $4(\mathrm{~A})$ shows the maximum rotation rate $(d \phi / d B)_{\max }$ as a function of intensity for the two different beam diameters used above. The inset shows the density for which the maximum rotation rate is reached as a function of intensity. We can relate the maximum rotation rate to magnetic field sensitivity using Eq. 

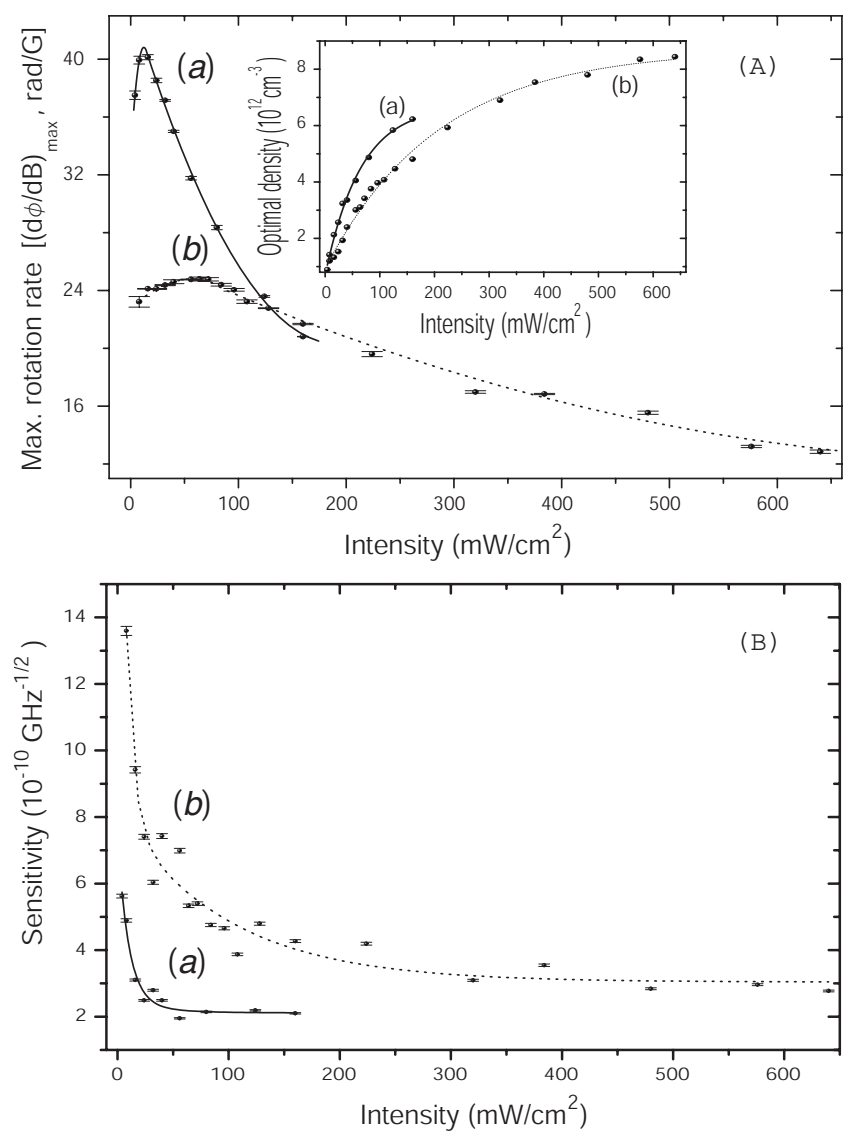

FIG. 4. (A) Maximum rotation rate as a function of laser intensity. The inset shows the density corresponding to the maximum rotation rate. Curve $(a)$ is for $d=4 \mathrm{~mm}$ beam diameter and curve $(b)$ is for $d=2 \mathrm{~mm}$. (B) Calculated sensitivity for the maximum rotation rate data. The dotted and dashed lines are presented only to guide the eye.

(2). This calculated sensitivity is shown in Fig. 4(B). We see that the sensitivity improves (drops) as the intensity is increased, but reaches a limiting value. The limiting value is improved by increasing the beam diameter and hence increasing the interaction time of the atoms with the laser. It is important to note that each point (with different intensity) is measured at the density for which the rotation rate is maximum.

These data demonstrate the interplay of an increasing rotation rate and increased transmission on the sensitivity calculated by Eq. (2). We find that the maximum rotation rates for different intensities are observed when the transmission is around $2-5 \%$. However, the density that provides the optimal sensitivity corresponds to a much higher transmission. This is shown in Fig. 5(A) for the rotation data with $I$ $=64 \mathrm{~mW} / \mathrm{cm}^{2}$ and diameter $d=2 \mathrm{~mm}$. The inset shows the transmission for this same condition. We clearly see that the optimal sensitivity does not occur at the same point as the maximum rotation rate. In other words, the two factors of Eq. (2) optimize at different densities. For our data, the optimal sensitivity occurs on the order of $\sim 50 \%$ transmission. Figure 5(B) shows the sensitivity derived for different intensities at their optimal densities. We find that the optimal sensitivity initially improves (drops) as the intensity increases,
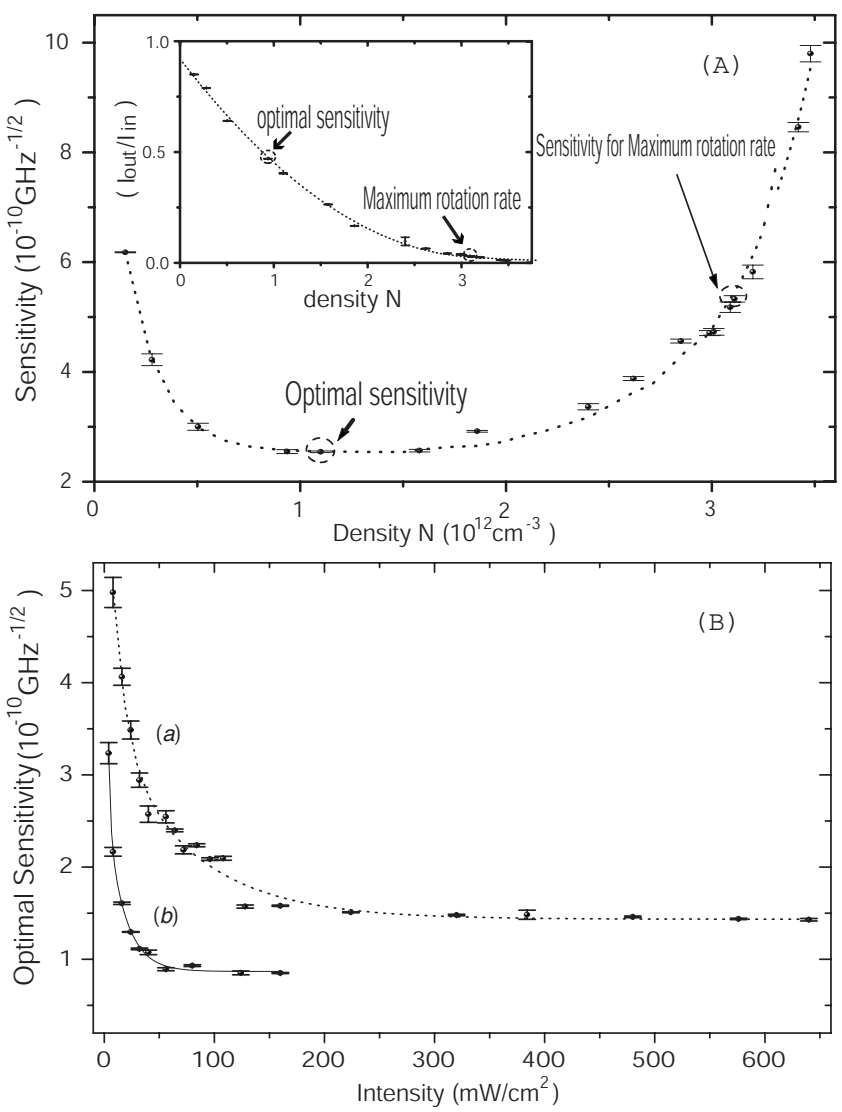

FIG. 5. (A) Sensitivity as a function of density for intensity $I$ $=64 \mathrm{~mW} / \mathrm{cm}^{2}$ and diameter $d=2 \mathrm{~mm}$. The plot shows that the optima sensitivity does not occur at the same density as for the maximum rotation rate. The inset shows the transmission $\left(I_{\text {out }} / I_{\text {in }}\right)$ versus density. (B) Optimal sensitivity for each intensity. Data set $(a)$ is for the $d=2 \mathrm{~mm}$ beam diameter and $(b)$ is for $d=4 \mathrm{~mm}$. The dotted and solid lines are presented only to guide the eye.

but then it reaches a limiting value. This behavior is similar to the case for the sensitivity corresponding to the maximum rotation rate, which is shown in Fig. 4(B). Figure 5(B) also shows that a larger laser beam diameter and corresponding smaller dephasing rate produces a better limiting value for the optimal sensitivity.

\section{CALCULATION OF ROTATION RATE AND SENSITIVITY}

In this section we theoretically investigate the saturation of the rotation rate $\partial \phi / \partial B$ and also the sensitivity $\delta B_{z}$. We consider two monochromatic fields with field polarizations $\hat{\epsilon}_{ \pm}$

$$
\vec{E}_{ \pm}(z, t)=\hat{\epsilon}_{ \pm} \mathcal{E}_{ \pm}(z) e^{i k_{ \pm} z-i \omega_{ \pm} t}+\text { c.c. },
$$

propagating along the $z$ direction inside a medium consisting of atoms having a three-level scheme as shown in Fig. 1. Here $\hat{\boldsymbol{\epsilon}}_{ \pm}$is the unit polarization vector corresponding to $\sigma_{ \pm}$ polarization, $\mathcal{E}_{\alpha}(z)$ is the field amplitude, and $k_{\alpha}$ corresponds to the propagation constant with central frequency $\omega_{\alpha}$, where $\alpha \rightarrow \pm$. The propagation of the field along the $z$ direction in 
the medium is governed by the Maxwell-Bloch equation

$$
\frac{\partial^{2} \vec{E}_{ \pm}}{\partial z^{2}}-\frac{1}{c^{2}} \frac{\partial^{2} \vec{E}_{ \pm}}{\partial t^{2}}=\frac{1}{\epsilon_{0} c^{2}} \frac{\partial^{2} \vec{P}_{ \pm}}{\partial t^{2}} .
$$

Assuming the same phase dependence for the polarization of the medium $\vec{P}_{ \pm}=\hat{\epsilon}_{ \pm} \mathcal{P}_{ \pm} e^{i k_{ \pm} z-i \omega_{ \pm} t}+$ c.c. as that for the field $\vec{E}$ given in Eq. (3), and using the slowly varying amplitude and phase approximations, we find [20]

$$
\frac{\partial \mathcal{E}_{ \pm}}{\partial z}=\frac{i k_{ \pm}}{2 \epsilon_{0}} \mathcal{P}_{ \pm} .
$$

Here, the atomic polarization $\mathcal{P}_{ \pm}=N \wp_{ \pm} \rho_{ \pm}\left(\wp_{+}=\wp_{a c}, \wp_{-}\right.$ $=\wp_{a b}, \rho_{+}=\rho_{a c}$, and $\left.\rho_{-}=\rho_{a b}\right), \wp_{\alpha}$ is the dipole moment, $\rho_{\alpha}$ corresponds to the density matrix element, $N$ is the atomic density, and $\alpha \rightarrow \pm$. Defining the Rabi frequencies due to the atom-field interaction $\Omega_{c}=\wp_{+} \mathcal{E}_{+} / 2 \hbar, \Omega_{b}=\wp_{-} \mathcal{E}_{-} / 2 \hbar$, and the above propagation equations can be written as

$$
\begin{aligned}
& \frac{\partial \Omega_{c}}{\partial z}=i \eta_{c} \rho_{a c}, \\
& \frac{\partial \Omega_{b}}{\partial z}=i \eta_{b} \rho_{a b},
\end{aligned}
$$

where $\eta_{b}=k_{-} N \wp_{-}^{2} /\left(\hbar \epsilon_{0}\right), \eta_{c}=k_{+} N \wp_{+}^{2} /\left(\hbar \epsilon_{0}\right)$. The equations for the density matrix elements of the three-level lambda system under consideration are

$$
\begin{gathered}
\frac{\partial \rho_{a a}}{\partial t}=-2\left(\gamma_{1}+\gamma_{2}\right) \rho_{a a}+i \Omega_{b} \rho_{b a}-i \Omega_{b}^{*} \rho_{a b}+i \Omega_{c} \rho_{c a}-i \Omega_{c}^{*} \rho_{a c}, \\
\frac{\partial \rho_{a b}}{\partial t}=-\left(\gamma_{1}+\gamma_{2}+i \delta_{1}\right) \rho_{a b}-i \Omega_{b}\left(\rho_{a a}-\rho_{b b}\right)+i \Omega_{c} \rho_{c b}, \\
\frac{\partial \rho_{a c}}{\partial t}=-\left(\gamma_{1}+\gamma_{2}+i \delta_{2}\right) \rho_{a c}+i \Omega_{b} \rho_{b c}-i \Omega_{c}\left(\rho_{a a}-\rho_{c c}\right), \\
\frac{\partial \rho_{b b}}{\partial t}=2 \gamma_{1} \rho_{a a}-i \Omega_{b} \rho_{b a}+i \Omega_{b}^{*} \rho_{a b}, \\
\frac{\partial \rho_{b c}}{\partial t}=-\left[\gamma_{0}+i\left(\delta_{2}-\delta_{1}\right)\right] \rho_{b c}+i \Omega_{b}^{*} \rho_{a c}-i \Omega_{c} \rho_{b a}, \\
\frac{\partial \rho_{c c}}{\partial t}=2 \gamma_{2} \rho_{a a}-i \Omega_{c} \rho_{c a}+i \Omega_{c}^{*} \rho_{a c},
\end{gathered}
$$

where $2 \gamma_{1}$ and $2 \gamma_{2}$ are the rates of spontaneous decay from the excited state $|a\rangle, \gamma_{0}$ is the ground-state dephasing rate, and $\delta_{1}$ and $\delta_{2}$ are the detunings of the $E_{-}$and $E_{+}$fields, respectively. The conservation of population gives $\rho_{a a}+\rho_{b b}$ $+\rho_{c c}=1$.

The solutions of Eqs. (6) and (7), along with the solutions of $\rho_{a b}$ and $\rho_{a c}$ obtained from Eqs. (8), describe the spatial evolution of the different polarization components of the fields inside the medium. Our numerical results focus on how the laser intensity and medium density affect the rotation rates and sensitivities. In Fig. 6(A), we show that the
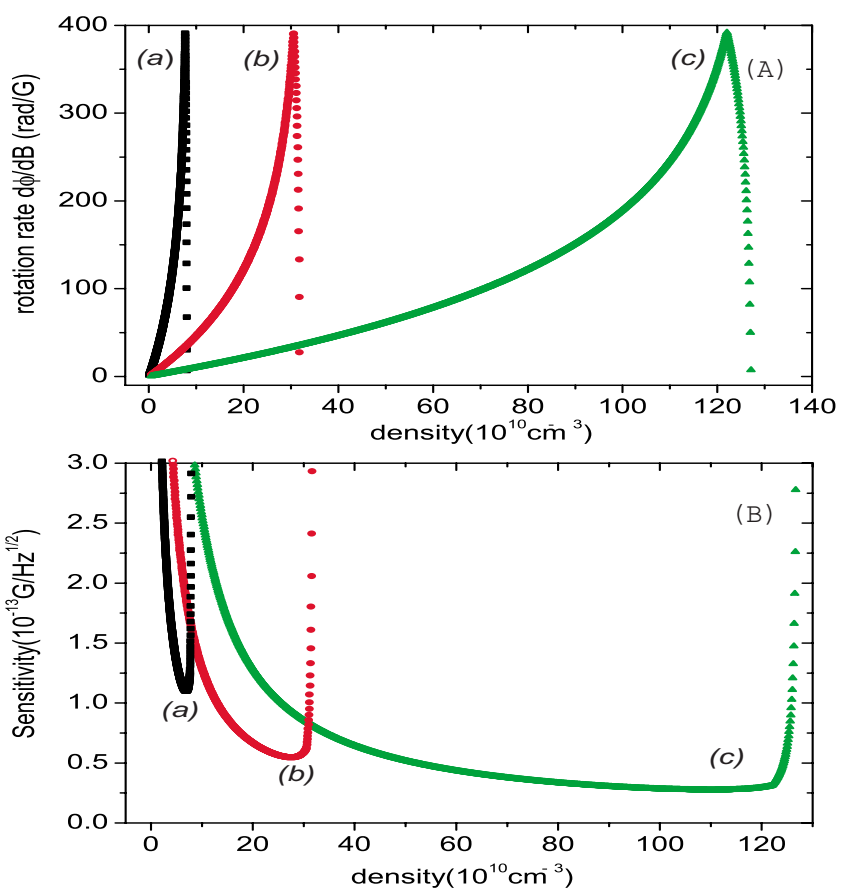

FIG. 6. (Color online) (A) Plot of rotation slope vs density for different laser Rabi frequency $|\Omega|$. Here $\gamma_{0}=0.001 \gamma$, and $\Omega_{b}=\Omega_{c}$, $\Omega=\Omega_{b}+\Omega_{c}$. (a) Squares: $|\Omega|=2 \gamma(b)$ Circles: $|\Omega|=4 \gamma$. (c) Triangles: $|\Omega|=8 \gamma$. (B) Sensitivity corresponding to the same data as in (c).

rotation rates for different Rabi frequencies increase with atomic density and the behavior of curves agree well with the analytical solution from Refs. [9,13]. Our numerical results also qualitatively agree with the experimental data shown in Fig. 3. The corresponding sensitivity derived from the data presented in Fig. 6(A) shows that higher density and intensity improve the sensitivity as depicted in Fig. 6(B). However, experimentally we observed that sensitivity has an upper limit in this NLMOR configuration, which could not be observed in our numerical calculation. That is because the above calculation assumes that the medium polarization has a linear dependence on the density of atoms, which becomes invalid at higher densities. Thus numerical results presented above perfectly describe the rotation rate and sensitivity for low density atomic gas cells.

\section{LIMIT OF SENSITIVITY IN HIGH-DENSITY REGIME}

At higher atomic densities, a strong incoherent process via incoherent emission and reabsorption of photons inside the medium known as radiation trapping $[18,21,22]$ plays a very dominant role in determining the atomic dynamics and the field propagation through the dense medium. Thus it becomes extremely difficult to deterministically calculate the atomic polarization and hence the polarization rotation rate, which strongly deviate from the linear dependence on density. The effect of density on resonant magneto-optical rotation in a relatively low-density medium has been discussed by Pustelny et al. in Ref. [23]. We use the phenomenological model, suggested by Matsko et al., to describe the radiation 


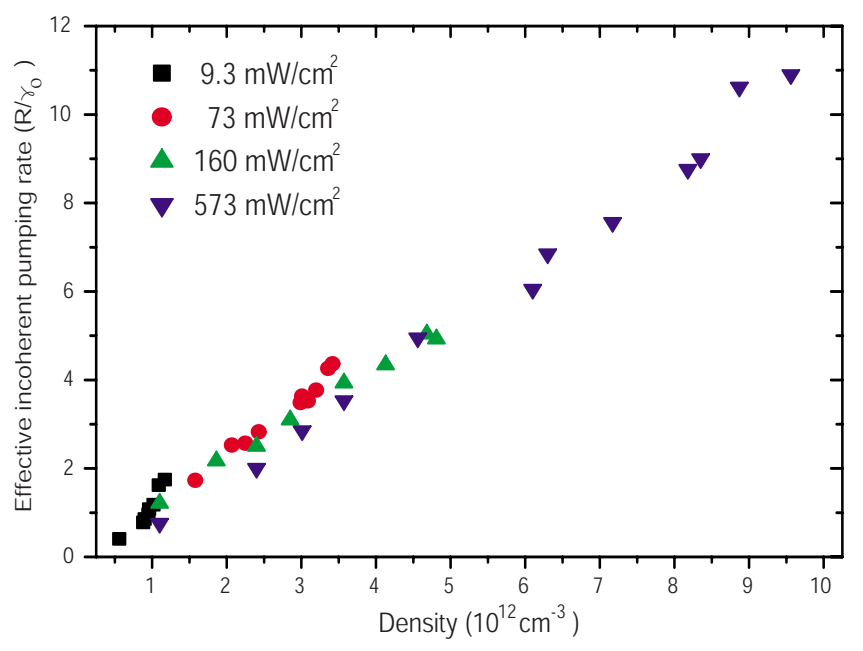

FIG. 7. (Color online) The scaled incoherent pumping rate $R / \gamma_{0}$ for laser beam diameter $d=2 \mathrm{~mm}$.

trapping via an incoherent pumping in a high-density medium. The rotation rate is given as [18]

$$
\left.\frac{d \phi(z)}{d B_{z}}\right|_{B_{z} \rightarrow 0}=\frac{2 \mu_{B}}{\hbar\left(\gamma_{0}+R\right)} \ln \left|\frac{I_{\text {in }}}{I_{\text {out }}}\right|,
$$

where $R$ is the incoherent pumping rate, $I_{\text {in }} / I_{\text {out }}$ is the inverse of the transmission rate, $\mu_{B}$ is the Bohr magneton, and $\gamma_{0}$ is the dephasing rate of ground-state Zeeman coherence. The incoherent pumping rate $R$ can be determined from measuring the rotation rate $d \phi / d B_{z}$, shown in Fig. 7. The measured incoherent pumping rate shows a linear dependence on the increase of atomic density. This holds for a very large range of intensity and density. It is observed that for $d=2 \mathrm{~mm}$ beam diameter, the incoherent pumping exceeds even the ground-state dephasing rate $\gamma_{0} \sim 20 \mathrm{kHz}$ at a density of 0.8 $\times 10^{12} \mathrm{~cm}^{-3}$. Another possible source of dephasing, such as spin exchange collision, is two orders of magnitude smaller than the decoherence caused by time-of-flight consideration [18].

We believe such an enhanced incoherent process, which also increases the effective ground-state dephasing, offers an explanation to the observed saturation of the magnetometer sensitivity. Following is a short physical explanation: At low atomic density, where transmission of the field is fairly high $\left(I_{\text {out }} / I_{\text {in }}>60 \%\right)$, an increase in contribution from rotation rate $\left(d \phi / d B_{z}\right)$ with the atomic density compensates the corresponding decrease in the square root of the number of transmitted photons $\sqrt{N_{p h}}$. This leads to increased sensitivity $\delta B_{z}$ with increased density. However, once the atomic density reaches a limit where radiation trapping becomes significant, both the rotation rate $\left(d \phi / d B_{z}\right)$ and field transmission $\left(\sqrt{N_{p h}}\right)$ suffer due to increased ground-state dephasing and absorption. Hence the sensitivity $\delta B_{z}$ saturates, as shown in Fig. 5.

\section{SUMMARY}

We have reported an experimental study of the nonlinear Faraday effect for relatively high input laser intensity and varying density. We have shown the behavior of the rotation rate for larger intensities and densities from the linear region $(\Omega \leqslant \gamma)$ to the nonlinear region $(\Omega \gg \gamma)$ for the first time. We have experimentally demonstrated that the optimal sensitivity saturates. We have also presented an extensive study on the behavior of the maximum rotation rate at different intensities and the corresponding sensitivity. Our numerical results for rotation rate and corresponding sensitivity qualitatively agree with the experimental results at lower atomic densities. The saturation of optimal sensitivity at higher densities are explained numerically by including phenomenological incoherent pumping that takes into account the radiation trapping at such densities. To date, the limitation of sensitivity via increasing the laser intensity and density has never been fully explored, so our experimental results could be very useful for choosing the optimal laser intensity and density for CPT-based magnetometer.

\section{ACKNOWLEDGMENTS}

The authors gratefully acknowledge useful discussions with M. Scully, D. Budker, I. Novikova, Y. Rostovtsev, A. Zhang, and J. Musser.
[1] J. M. Geremia, J. K. Stockton, and H. Mabuchi, Phys. Rev. Lett. 94, 203002 (2005).

[2] V. Petersen, L. B. Madsen, and K. Molmer, Phys. Rev. A 71, 012312 (2005).

[3] E. Taskova, S. Gateva, E. Alipeva, K. Kowalski, M. Glodz, and J. Szonert, Appl. Opt. 43, 4178 (2004).

[4] E. B. Alexandrov, M. V. Balabas, A. S. Pasgalev, A. K. Vershovskii, and N. N. Yakobson, Laser Phys. 6, 244 (1996).

[5] E. B. Alexandrov and V. A. Bonch-Bruevich, Opt. Eng. (Bellingham) 31, 711 (1992).

[6] D. Budker, D. F. Kimball, S. M. Rochester, V. V. Yashchuk, and M. Zolotorev, Phys. Rev. A 62, 043403 (2000).
[7] W. Happer, Rev. Mod. Phys. 44, 169 (1972).

[8] M. O. Scully, Phys. Rev. Lett. 67, 1855 (1991).

[9] M. Fleischhauer, A. B. Matsko, and M. O. Scully, Phys. Rev. A 62, 013808 (2000).

[10] E. Arimondo, in Progress in Optics, edited by E. Wolf (Elsevier Science, Amsterdam, 1996), Vol. XXXV, p. 257; S. E. Harris, Phys. Today 50, 36 (1997); J. P. Marangos, J. Mod. Opt. 45, 471 (1998).

[11] M. O. Scully, Phys. Rev. Lett. 67, 1855 (1991); A. S. Zibrov, M. D. Lukin, L. W. Hollberg, D. E. Nikonov, M. O. Scully, H. G. Robinson, and V. L. Velichansky, ibid. 76, 3935 (1996).

[12] L. V. Hau, S. E. Harris, Z. Dutton, and C. H. Behroozi, Nature 
(London) 397, 594 (1999); M. M. Kash, V. A. Sautenkov, A. S. Zibrov, L. Hollberg, G. R. Welch, M. D. Lukin, Y. Rostovtsev, E. S. Fry, and M. O. Scully, Phys. Rev. Lett. 82, 5229 (1999); D. Budker, D. F. Kimball, S. M. Rochester, and V. V. Yashchuk, ibid. 83, 1767 (1999).

[13] V. A. Sautenkov, M. D. Lukin, C. J. Bednar, I. Novikova, E. Mikhailov, M. Fleischhauer, V. L. Velichansky, G. R. Welch, and M. O. Scully, Phys. Rev. A 62, 023810 (2000).

[14] I. Novikova and G. R. Welch, J. Mod. Opt. 49, 349 (2002).

[15] I. K. Kominis, T. W. Kornack, J. C. Allred, and M. V. Romalis, Nature (London) 422, 596 (2003).

[16] W. Gawlik, L. Krzemien, S. Pustelny, D. Sang, J. Zachorowski, M. Graf, A. O. Sushkov, and D. Budker, Appl. Phys. Lett. 88, 131108 (2006).
[17] I. Novikova, A. B. Matsko, V. L. Velichansky, M. O. Scully, and G. R. Welch, Phys. Rev. A 63, 063802 (2001).

[18] A. B. Matsko, I. Novikova, M. O. Scully, and G. R. Welch, Phys. Rev. Lett. 87, 133601 (2001).

[19] A. K. Patnaik, P. S. Hsu, G. S. Agarwal, G. R. Welch, and M. O. Scully, Phys. Rev. A 75, 023807 (2007).

[20] A. K. Patnaik and G. S. Agarwal, Opt. Commun. 179, 97 (2000); 199, 127 (2001).

[21] A. B. Matsko, I. Novikova, and G. R. Welch, J. Mod. Opt. 49, 367 (2002).

[22] G. Ankerhold, M. Schiffer, D. Mutschall, T. Scholz, and W. Lange, Phys. Rev. A 48, R4031 (1993).

[23] S. Pustelny, W. Lewoczko, and W. Gawlik, J. Opt. Soc. Am. B 22, 37 (2005). 